Journal of the Operations Research Society of Japan

Vol. 43, No. 1, March 2000

\title{
ITERATIVE METHODS FOR APPROXIMATION OF FIXED POINTS AND THEIR APPLICATIONS
}

\author{
Wataru Takahashi \\ Tokyo Institute of Technology
}

(Received December 15, 1998; Revised May 11, 1999)

\begin{abstract}
In this article, we deal with iterative methods for approximation of fixed points and their applications. We first discuss fixed point theorems for a nonexpansive mapping or a family of nonexpansive mappings. In particular, we state a fixed point theorem which answered affirmatively a problem posed during the Conference on Fixed Point Theory and Applications held at CIRM, Marseille-Luminy, 1989. Then we discuss nonlinear ergodic theorems of Baillon's type for nonlinear semigroups of nonexpansive mappings. In particular, we state nonlinear ergodic theorems which answered affirmatively the problem posed during the Second World Congress on Nonlinear Analysts, Athens, Greece, 1996. Next, we deal with weak and strong convergence theorems of Mann's type and Halpern's type in a Banach space. Finally, using these results, we consider the feasibility problem by convex combinations of nonexpansive retractions and the convex minimization problem of finding a minimizer of a convex function.
\end{abstract}

\section{Introduction}

Let $C$ be a nonempty closed convex subset of a real Hilbert space $H$ and let $f$ be a proper convex lower semicontinuous function of $H$ into $(-\infty, \infty]$. Consider a convex minimization problem

$$
\min \{f(x): x \in C\}=\alpha \text {. }
$$

The number $\alpha$ is called an optimal value, $C$ is called an admissible set and $M=\{y \in C$ : $f(y)=\alpha\}$ is called an optimal set. Next, define a function $g: H \rightarrow(-\infty, \infty]$ as follows:

$$
g(x)= \begin{cases}f(x), & x \in C, \\ \infty, & x \notin C .\end{cases}
$$

Then, $g$ is a proper lower semicontinuous convex function of $H$ into $(-\infty, \infty]$. So, we consider the convex minimization problem

$$
\min \{g(x): x \in H\}
$$

where $g$ is a proper lower semicontinuous convex function of $H$ into $(-\infty, \infty]$. For such a $g$, we can define a multivalued operator $\partial g$ on $H$ by

$$
\partial g(x)=\left\{x^{*} \in H: g(y) \geqq g(x)+\left(x^{*}, y-x\right), y \in H\right\}
$$

for all $x \in H$. Such a $\partial g$ is said to be the subdifferential of $g$. Let $C$ be a nonempty closed convex subset of a real Hibert space $H$. Then a mapping $T: C \rightarrow C$ is called nonexpansive on $C$ if

$$
\|T x-T y\| \leqq\|x-y\| \quad \text { for all } x, y \in C
$$


We denote by $F(T)$ the set of fixed point of $T$. Let $A \subset H \times H$. Then, we can define a multivalued operator $B$ from $H$ to $H$ by

$$
B x=\{y \in H:(x, y) \in A\}
$$

for all $x \in H$. Inversely, if $B$ is a multivalued operator from $H$ to $H$, then we can define a set $A$ in $H \times H$ by $A=\{(x, y): x \in H, y \in B x\}$. So, it is natural to regard a set in $H \times H$ in the same light with a multivalued operator from $H$ to $H$. Let $A \subset H \times H$. Then, we define the domain of $A$ and the range of $A$ as follows:

$$
\begin{aligned}
& D(A)=\{x \in E: A x \neq \phi\} \\
& R(A)=\cup\{A x: x \in D(A)\} .
\end{aligned}
$$

We also define a multivalued operator $A^{-1}$ from $H$ to $H$ by

$$
A^{-1} y=\{x \in H: y \in A x\}
$$

for all $y \in H$. From this definition, we have $x \in A^{-1} 0 \Leftrightarrow 0 \in A x$. An operator $A \subset H \times H$ is accretive if for $\left(x_{1}, y_{1}\right),\left(x_{2}, y_{2}\right) \in A$,

$$
\left(x_{1}-x_{2}, y_{1}-y_{2}\right) \geqq 0 .
$$

If $A$ is accretive, we can define, for each positive $\lambda$, the resolvent $J_{\lambda}: R(I+\lambda A) \rightarrow D(A)$ by $J_{\lambda}=(I+\lambda A)^{-1}$. We know that $J_{\lambda}$ is a nonexpansive mapping. An accretive operator $A \subset H \times H$ is called $m$-accretive if $R(I+\lambda A)=H$ for all $\lambda>0$. If $g: H \rightarrow(-\infty, \infty]$ is a proper lower semicontinuous convex function, then $\partial g$ is an $\mathrm{m}$-accretive operator. For an m-accretive operator $A$, we can consider the following initial value problem:

$$
\begin{gathered}
\frac{d u(t)}{d t}+A u(t) \ni 0, \quad t>0, \\
u(0)=x,
\end{gathered}
$$

where $x$ is an element of $\overline{D(A)}$. Then, it is well known that $(* *)$ has a unique strong solution $u:[0, \infty) \rightarrow H$. Putting $S(t) x=u(t)$, we know that the family $\{S(t): t \in[0, \infty)\}$ of mappings on $\overline{D(A)}$ satisfies the following conditions:

(i) $S(t+s) x=S(t) S(s) x$ for every $t, s \in[0, \infty)$ and $x \in \overline{D(A)}$;

(ii) $S(0) x=x$ for every $x \in \overline{D(A)}$;

(iii) for each $x \in \overline{D(A)}, t \mapsto S(t) x$ is continuous;

(iv) $\|S(t) x-S(t) y\| \leqq\|x-y\|$ for every $x, y \in \overline{D(A)}$ and $t \in[0, \infty)$.

Such a family $\{S(t): t \in[0, \infty)\}$ is called a one-parameter nonexpansive semigroup on $\overline{D(A)}$; see Brézis [7]. We also know that

$$
\begin{aligned}
0 \in \partial g\left(x_{0}\right) & \Leftrightarrow g\left(x_{0}\right)=\min \{g(x): x \in H\} \\
& \Leftrightarrow x_{0} \in \bigcap_{t \geqq 0} F(S(t)),
\end{aligned}
$$

where $F(S(t))$ is the set of fixed points of $S(t)$. Further, we have that for $\lambda>0$,

$$
0 \in \partial g\left(x_{0}\right) \Leftrightarrow J_{\lambda} x_{0}=x_{0} .
$$


Thus, a convex minimization problem is equivalent to a fixed point problem for a nonexpansive mapping or a family of nonexpansive mappings. Further, we know that one method for solving (*) is the proximal point algorithm first introduced by Martinet [43]. The proximal point algorithm is based on the notion of resolvent $J_{\lambda}$, i.e.,

$$
J_{\lambda} x=\arg \min \left\{g(z)+\frac{1}{2 \lambda}\|z-x\|^{2}: z \in H\right\}
$$

introduced by Moreau [47]. The proximal point algorithm is an iterative procedure, which starts at a point $x_{1} \in H$, and generates recursively a sequence $\left\{x_{n}\right\}$ of points $x_{n+1}=J_{\lambda_{n}} x_{n}$, where $\left\{\lambda_{n}\right\}$ is a sequence of positive numbers; see, for instance, Rockafellar [52]. On the other hand, let $\left\{g_{1}, g_{2}, \ldots, g_{n}\right\}$ be a finite family of real valued continuous convex functions on a Hilbert space $H$. The problem is to find a solution of the finite convex inequality system, i.e., to find such a point $x \in C$ that

$$
C=\left\{x \in H: g_{i}(x) \leqq 0, i=1,2, \ldots, n\right\}
$$

Such a problem is called the feasibility problem. This problem is also connected with approximation of fixed points.

In this article, we first discuss fixed point theorems for a nonexpansive mapping or a family of nonexpansive mappings. In particular, we state a fixed point theorem which answered affirmatively a problem [34] posed during the Conference on Fixed Point Theory and Applications held at CIRM, Marseille-Luminy, 1989. Then we discuss nonlinear ergodic theorems of Baillon's type for nonlinear semigroups of nonexpansive mappings. In particular, we state nonlinear ergodic theorems which answered affirmatively the problem [69] posed during the Second World Congress on Nonlinear Analysts, Athens, Greece, 1996. Next, we deal with weak and strong convergence theorems of Mann's type and Halpern's type in a Banach space. Finally, using these results, we consider the feasibility problem by convex combinations of nonexpansive retractions and the convex minimization problem of finding a minimizer of a convex function.

\section{Preliminaries}

Let $C$ be a nonempty closed convex subset of a Banach space $E$ and let $T$ be a mapping of $C$ into $C$. Then we denote by $R(T)$ the range of $T$. A mapping $T$ of $C$ into $C$ is said to be asymptotically regular if for every $x \in C, T^{n} x-T^{n+1} x$ converges to 0 . Let $D$ be a subset of $C$ and let $P$ be a mapping of $C$ into $D$. Then $P$ is said to be sunny if

$$
P(P x+t(x-P x))=P x
$$

whenever $P x+t(x-P x) \in C$ for $x \in C$ and $t \geq 0$. A mapping $P$ of $C$ into $C$ is said to be a retraction if $P^{2}=P$. If a mapping $P$ of $C$ into $C$ is a retraction, then $P z=z$ for every $z \in R(P)$. A subset $D$ of $C$ is said to be a sunny nonexpansive retract of $C$ if there exists a sunny nonexpansive retraction of $C$ onto $D$.

Let $E$ be a Banach space. Then, for every $\varepsilon$ with $0 \leq \varepsilon \leq 2$, the modulus $\delta(\varepsilon)$ of convexity of $E$ is defined by

$$
\delta(\varepsilon)=\inf \left\{1-\left\|\frac{x+y}{2}\right\|:\|x\| \leq 1,\|y\| \leq 1,\|x-y\| \geq \varepsilon\right\}
$$

A Banach space $E$ is said to be uniformly convex if $\delta(\varepsilon)>0$ for every $\varepsilon>0$. $E$ is also said to be strictly convex if $\|x+y\|<2$ for $x, y \in E$ with $\|x\| \leq 1,\|y\| \leq 1$ and $x \neq y$. A uniformly convex Banach space is strictly convex. 
Let $E$ be a Banach space and let $E^{*}$ be its dual, that is, the space of all continuous linear functionals $x^{*}$ on $E$. The value of $x^{*} \in E^{*}$ at $x \in E$ will be denoted by $\left(x, x^{*}\right)$. With each $x \in E$, we associate the set $J(x)=\left\{x^{*} \in E^{*}:\left(x, x^{*}\right)=\|x\|^{2}=\left\|x^{*}\right\|^{2}\right\}$. Using the Hahn-Banach theorem, it is immediately clear that $J(x) \neq \phi$ for any $x \in E$. Then the multivalued operator $J: E \rightarrow E^{*}$ is called the duality mapping of $E$. Let $U=\{x \in E:\|x\|=1\}$ be the unit sphere of $E$. Then a Banach space $E$ is said to be smooth provided

$$
\lim _{t \rightarrow 0} \frac{\|x+t y\|-\|x\|}{t}
$$

exists for each $x, y \in U$. When this is the case, the norm of $E$ is said to be Gâteaux differentiable. It is said to be Fréchet differentiable if for each $x$ in $U$, this limit is attained uniformly for $y$ in $U$. The space $E$ is said to have a uniformly Gâteaux differentiable norm if for each $y \in U$, the limit is attained uniformly for $x \in U$. It is well known that if $E$ is smooth, then the duality mapping $J$ is single valued. It is also known that if $E$ has a Fréchet differentiable norm, then $J$ is norm to norm continuous; see [17] for more details. A closed convex subset $C$ of a Banach space $E$ is said to have normal structure if for each closed bounded convex subset $K$ of $C$, which contains at least two points, there exists an element of $K$ which is not a diametral point of $K$. Baillon and Schöneberg [6] also introduced the following weakening of the concept of normal structure: A closed convex subset $C$ of a Banach space is said to have asymptotic normal structure if for each closed bounded convex subset $K$ of $C$, which contains at least two points and each sequence $\left\{x_{n}\right\}$ in $K$ satisfying $x_{n}-x_{n+1} \rightarrow 0$ as $n \rightarrow \infty$, there is a point $x \in K$ such that $\liminf _{n \rightarrow \infty}\left\|x_{n}-x\right\|<\delta(K)$, where $\delta(K)$ is the diameter of $K$. It is well known that a closed convex subset of a uniformly convex Banach space has normal structure and a compact convex subset of a Banach space has normal structure. A Banach space $E$ is said to satisfy Opial's condition [48] if $x_{n} \rightarrow x$ and $x \neq y$ imply

$$
\liminf _{n \rightarrow \infty}\left\|x_{n}-x\right\|<\liminf _{n \rightarrow \infty}\left\|x_{n}-y\right\|,
$$

where $\rightarrow$ denotes the weak convergence to $x$. Let $S$ be a semitopological semigroup, i.e., a semigroup with Hausdorff topology such that for each $s \in S$, the mappings $t \mapsto t s$ and $t \mapsto s t$ of $S$ into itself are continuous. Let $B(S)$ be the Banach space of all bounded real valued functions on $S$ with supremum norm and let $X$ be a subspace of $B(S)$ containing constants. Then, an element $\mu$ of $X^{*}$ is called a mean on $X$ if $\|\mu\|=\mu(1)=1$. We know that $\mu \in X^{*}$ is a mean on $X$ if and only if

$$
\inf \{f(s): s \in S\} \leq \mu(f) \leq \sup \{f(s): s \in S\}
$$

for every $f \in X$. A real valued function $\mu$ on $X$ is called a submean on $X$ if the following properties are satisfied:

(i) $\mu(f+g) \leq \mu(f)+\mu(g)$ for every $f, g \in X$;

(ii) $\mu(\alpha f)=\alpha \mu(f)$ for every $f \in X$ and $\alpha \geq 0$;

(iii) for $f, g \in X, f \leq g$ implies $\mu(f) \leq \mu(g)$;

(iv) $\mu(c)=c$ for every constant function $c$.

Clearly every mean on $X$ is a submean. The notion of submean was first introduced by Mizoguchi and Takahashi [46]. For a submean $\mu$ on $X$ and $f \in X$, sometimes we use $\mu_{t}(f(t))$ instead of $\mu(f)$. For each $s \in S$ and $f \in B(S)$, we define elements $\ell_{s} f$ and $r_{s} f$ of $B(S)$ given by $\left(\ell_{s} f\right)(t)=f(s t)$ and $\left(r_{s} f\right)(t)=f(t s)$ for all $t \in S$. Let $X$ be a subspace of $B(S)$ containing constants which is invariant under $\ell_{s}, s \in S$ (resp. $r_{s}, r \in S$ ). Then a mean $\mu$ on 
$X$ is said to be left invariant (resp. right invariant) if $\mu(f)=\mu\left(\ell_{s} f\right) \quad\left(\operatorname{resp} . \mu(f)=\mu\left(r_{s} f\right)\right)$ for all $f \in X$ and $s \in S$. An invariant mean is a left and right invariant mean. A submean $\mu$ on $X$ is said to be left subinvariant if $\mu(f) \leq \mu\left(\ell_{s} f\right)$ for all $f \in X$ and $s \in S$. Let $S$ be a semitopological semigroup. Then $S$ is called left (resp. right) reversible if any two closed right (resp. left) ideals of $S$ have non-void intersection. If $S$ is left reversible, $(S, \leq)$ is a directed system when the binary relation " $\leq$ " on $S$ is defined by $a \leq b$ if and only if $\{a\} \cup \overline{S a} \supset\{b\} \cup \overline{S b}, a, b \in S$. Similarly, we can define the binary relation " $\leq$ " on a right reversible semitopological semigroup $S$.

\section{Fixed Point Theorems}

In this section, we discuss fixed point theorems for a nonexpansive mapping or a family of nonexpansive mappings. The first fixed point theorem for nonexpansive mappings was established in 1965 by Browder [8]. He proved that if $C$ is a bounded closed convex subset of a Hilbert space $H$ and $T$ is a nonexpansive mapping of $C$ into itself, then $T$ has a fixed point in $C$. Almost immediately, both Browder [9] and Göhde [20] proved that the same is true if $E$ is a uniformly convex Banach space. Kirk [31] also proved the following theorem:

Theorem 3.1 ([31]) Let $E$ be a reflexive Banach space and let $C$ be a nonempty bounded closed convex subset of $E$ which has normal structure. Let $T$ be a nonexpansive mapping of $C$ into itself. Then $F(T)$ is nonempty.

After kirk's theorem, many fixed point theorems concerning nonexpansive mappings have been proved in a Hilbert space or a Banach space. In particular, Baillon and Schöneberg [6] introduced the concept of asymptotic normal structure and generalized Kirk's fixed point theorem as follows:

Theorem 3.2 ([6]) Let $E$ be a reflexive Banach space and let $C$ be a nonempty bounded closed convex subset of $E$ which has asymptotic normal structure. Let $T$ be a nonexpansive mapping of $C$ into itself. Then $F(T)$ is nonempty.

On the other hand, DeMarr [16] proved the following fixed point theorem for a commutative family of nonexpansive mappings.

Theorem 3.3 ([16]) Let $C$ be a compact convex subset of a Banach space $E$ and let $\mathcal{S}$ be a commutative family of nonexpansive mappings of $C$ into itself. Then $\mathcal{S}$ has a common fixed point in $C$, i.e., there exists $z \in C$ such that $T z=z$ for every $T \in \mathcal{S}$.

Browder [9] proved the following fixed point theorem without compactness:

Theorem 3.4 ([9]) Let $C$ be a bounded closed convex subset of a uniformly convex Banach space $E$ and let $\mathcal{S}$ be a commutative family of nonexpansive mappings of $C$ into itself. Then $\mathcal{S}$ has a common fixed point in $C$

Further, we try to extend these theorems to a noncommutative semigroup of nonexpansive mappings. Let $S$ be a semitopological semigroup and let $C$ be a nonempty subset of a Banach space $E$. Then a family $\mathcal{S}=\left\{T_{s}: s \in S\right\}$ of mappings of $C$ into itself is called a nonexpansive semigroup on $C$ if it satisfies the following:

(i) $T_{s t} x=T_{s} T_{t} x$ for all $s, t \in S$ and $x \in C$;

(ii) for each $x \in C$, the mapping $s \mapsto T_{s} x$ is continuous;

(iii) for each $s \in S, T_{s}$ is a nonexpansive mapping of $C$ into itself.

For a nonexpansive semigroup $\mathcal{S}=\left\{T_{s}: s \in S\right\}$ on $C$, we denote by $F(\mathcal{S})$ the set of common fixed points of $T_{s}, s \in S$. Let $S$ be a semitopological semigroup, let $C(S)$ be the Banach space of all bounded continuous functions on $S$ and let $R U C(S)$ be the space of all bounded right uniformly continuous functions on $S$, i.e., all $f \in C(S)$ such that the 
mapping $s \mapsto r_{s} f$ is continuous. Then $R U C(S)$ is a closed subalgebra of $C(S)$ containing constants and invariant under $\ell_{s}$ and $r_{s}, s \in S$; see [44] for more details.

In 1969, Takahashi [62] proved the first fixed point theorem for a noncommutative semigroup of nonexpansive mappings which generalizes DeMarr's fixed point theorem, that is, he proved that any discrete left amenable semigroup has a common fixed point. Mitchell [45] generalized Takahashi's result by showing that any discrete left reversible semigroup has a common fixed point. Lau proved the following theorem in [33]:

Theorem 3.5 ([33]) Let $S$ be a semitopological semigroup and let $A(S)$ be the space of all $f \in C(S)$ such that $\left\{\ell_{s} f: s \in S\right\}$ is relatively compact in the norm topology of $C(S)$. Let $\mathcal{S}=\left\{T_{s}: s \in S\right\}$ be a nonexpansive semigroup on a compact convex subset $C$ of a Banach space $E$. Then $A(S)$ has a left invariant mean if and only if $\mathcal{S}$ has a common fixed point in $C$.

Lim [41] generalized Kirk's result [31], Browder's result [9] and Mitchell's result [45] by showing the following theorem:

Theorem 3.6 ([41]) Let $S$ be a left reversible semitopological semigroup. Let $C$ be a weakly compact convex subset of a Banach space $E$ which has normal structure and let $\mathcal{S}=\left\{T_{s}\right.$ : $s \in S\}$ be a nonexpansive semigroup on $C$. Then $\mathcal{S}$ has a common fixed point in $C$.

Takahashi and Jeong [71] also generalized Browder's result [9] by using the concept of submean; see also [79].

Theorem 3.7 ([71]) Let $S$ be a semitopological semigroup. Let $\mathcal{S}=\left\{T_{s}: s \in S\right\}$ be a nonexpansive semigroup on a bounded closed convex subset $C$ of a uniformly convex Banach space $E$. Suppose that $R U C(S)$ has a left subinvariant submean. Then $\mathcal{S}$ has a common fixed point in $C$.

To prove Theorem 3.7, we need the following lemma [81]:

Lemma $\mathbf{3 . 8}([\mathbf{8 1}])$ Let $p>1$ and $b>0$ be two fixed numbers. Then a Banach space $E$ is uniformly convex if and only if there exists a continuous, strictly increasing, and convex function (depending on $p$ and $b) g:[0, \infty) \rightarrow[0, \infty)$ such that $g(0)=0$ and

$$
\|\lambda x+(1-\lambda) y\|^{p} \leqq \lambda\|x\|^{p}+(1-\lambda)\|y\|^{p}-W_{p}(\lambda) g(\|x-y\|)
$$

for all $x, y \in B_{b}$ and $0 \leqq \lambda \leqq 1$, where $W_{p}(\lambda)=\lambda(1-\lambda)^{p}+\lambda^{p}(1-\lambda)$ and $B_{b}$ is the closed ball with radius $b$ and centered at the origin.

We may comment on the relationship between " $R U C(S)$ has an invariant mean" and " $S$ is left reversible". As well known, they do not imply each other in general. But if $R U C(S)$ has sufficiently many functions to separate closed sets, then "RUC $(S)$ has an invariant mean" would imply "S is left and right reversible". Recently, Lau and Takahashi [39] generalized Lim's result [41] and Takahashi and Jeong's result [71].

Theorem 3.9 ([39]) Let $S$ be a semitopological semigroup, let $C$ be a nonempty weakly compact convex subset of a Banach space $E$ which has normal structure and let $\mathcal{S}=\left\{T_{s}: s \in\right.$ $S\}$ be a nonexpansive semigroup on $C$. Suppose $R U C(S)$ has a left subinvariant submean. Then $\mathcal{S}$ has a common fixed point in $C$.

To prove Theorem 3.9, we need two lemmas.

Lemma 3.10 ([40]) A closed convex subset $C$ of a Banach space has normal structure if and only if it does not contain a sequence $\left\{x_{n}\right\}$ such that for some $c>0$,

$$
\left\|x_{n}-x_{m}\right\| \leqq c \text { and }\left\|x_{n+1}-\overline{x_{n}}\right\| \geqq c-\frac{1}{n^{2}}
$$

for all $n \geqq 1$ and $m \geqq 1$, where $\overline{x_{n}}=\frac{1}{n} \sum_{i=1}^{n} x_{i}$. 
Lemma 3.11 ([19]) Let $X$ be a compact convex subset of a separated topological vector space $E$, let $f_{1}, f_{2}, \ldots, f_{n}$ be a finite family of lower semicontinuous convex functions from $X$ into $R$ and let $c \in R$, where $R$ denotes the set of real numbers. Then the following conditions (i) and (ii) are equivalent:

(i) There exists $x_{0} \in X$ such that $f_{i}\left(x_{0}\right) \leqq c$ for all $i=1,2, \ldots, n$;

(ii) for any finite non-negative real numbers $\left\{\alpha_{1}, \alpha_{2}, \ldots, \alpha_{n}\right\}$ with $\sum_{i=1}^{n} \alpha_{i}=1$, there exists $y \in X$ such that $\sum_{i=1}^{n} \alpha_{i} f_{i}(y) \leqq c$.

Theorem 3.9 answers affirmatively a problem [34] posed during the Conference on Fixed Point Theory and Applications held at CIRM, Marseille-Luminy, 1989, whether Lim's result and Takahashi and Jeong's result can be fully extended to such Banach spaces for amenable semigroups. We do not know whether "normal structure "in Theorem 3.9 would be replaced by "asymptotic normal structure".

\section{Weak Convergence Theorems}

The first nonlinear ergodic theorem for nonexpansive mappings was established in 1975 by Baillon [4] in the framework of a Hilbert space.

Theorem 4.1 ([4]) Let $C$ be a closed convex subset of a Hilbert space $H$ and let $T$ be a nonexpansive mapping of $C$ into itself. If the set $F(T)$ of fixed points of $T$ is nonempty, then for each $x \in C$, the Cesàro means

$$
S_{n}(x)=\frac{1}{n} \sum_{k=0}^{n-1} T^{k} x
$$

converge weakly to some $y \in F(T)$.

This theorem was extended to a uniformly convex Banach space whose norm is Fréchet differentiable by Bruck [12].

Theorem 4.2 ([12]) Let $C$ be a closed convex subset of a uniformly convex Banach space $E$ with a Fréchet differentiable norm. If $T: C \rightarrow C$ is a nonexpansive mapping with a fixed point, then the Cesàro means of $\left\{T^{n} x\right\}$ converge weakly to a fixed point of $T$.

In their theorems, putting $y=P x$ for each $x \in C$, we have that $P$ is a nonexpansive retraction of $C$ onto $F(T)$ such that $P T^{n}=T^{n} P=P$ for all $n=1,2, \ldots$ and $P x \in$ $\overline{c o}\left\{T^{n} x: n=0,1,2, \ldots\right\}$ for each $x \in C$, where $\overline{c o} A$ is the closure of the convex hull of $A$. We discuss nonlinear ergodic theorems for a nonlinear semigroup of nonexpansive mappings in a Hilbert space or a Banach space. Before discussing them, we give a definition. Let $\left\{\mu_{\alpha}: \alpha \in A\right\}$ be a net of means on $R U C(S)$. Then $\left\{\mu_{\alpha} \in A\right\}$ is said to be asymptotically invariant if for each $f \in R U C(S)$ and $s \in S$,

$$
\mu_{\alpha}(f)-\mu_{\alpha}\left(\ell_{s} f\right) \rightarrow 0 \text { and } \mu_{\alpha}(f)-\mu_{\alpha}\left(r_{s} f\right) \rightarrow 0 \text {. }
$$

Let us give an example of asymptotically invariant nets. Let $S=\{0,1,2, \ldots\}$ and let $N$ be the set of positive integers. Then for $f=\left(x_{0}, x_{1}, \ldots\right) \in B(S)$ and $n \in N$, the real valued function $\mu_{n}$ defined by

$$
\mu_{n}(f)=\frac{1}{n} \sum_{k=0}^{n-1} x_{k}
$$


is a mean. Further since for $f=\left(x_{0}, x_{1}, \ldots\right) \in B(S)$ and $m \in N$

$$
\begin{aligned}
\left|\mu_{n}(f)-\mu_{n}\left(r_{m} f\right)\right| & =\left|\frac{1}{n} \sum_{k=0}^{n-1} x_{k}-\frac{1}{n} \sum_{k=0}^{n-1} x_{k+m}\right| \\
& \leq \frac{1}{n} \cdot 2 m\|f\| \rightarrow 0
\end{aligned}
$$

as $n \rightarrow \infty$ and $S$ is commutative, $\left\{\mu_{n}\right\}$ is an asymptotically invariant net of means.

If $C$ is a nonempty subset of a Hilbert space $H$ and $\mathcal{S}=\left\{T_{s}: s \in S\right\}$ is a nonexpansive semigroup on $C$ such that $\left\{T_{s} x: s \in S\right\}$ is bounded for some $x \in C$, then we know that for each $u \in C$ and $v \in H$, the functions $f(t)=\left\|T_{t} u-v\right\|^{2}$ and $g(t)=\left(T_{t} u, v\right)$ are in $R U C(S)$. Let $\mu$ be a mean on $R U C(S)$. Then since for each $x \in C$ and $y \in H$, the real valued function $t \mapsto\left(T_{t} x, y\right)$ is in $R U C(S)$, we can define the value $\mu_{t}\left(T_{t} x, y\right)$ of $\mu$ at this function. By linearity of $\mu$ and of the inner product, this is linear in $y$; moreover, since

$$
\left.\left|\mu_{t}\left(T_{t} x, y\right)\right| \leq\|\mu\| \cdot \sup _{t}\left|\left(T_{t} x, y\right)\right| \leq \sup _{t}\left\|T_{t} x\right\|\right) \cdot\|y\|,
$$

it is continuous in $y$. So, by the Riesz theorem, there exists an $x_{0} \in H$ such that

$$
\mu_{t}\left(T_{t} x, y\right)=\left(x_{0}, y\right)
$$

for every $y \in H$. We write such an $x_{0}$ by $T_{\mu} x$; see [64,67] for more details.

Now we can state a nonlinear ergodic theorem for noncommutative semigroups of nonexpansive mappings in a Hilbert space.

Theorem 4.3 ([68]) Let $C$ be a nonempty subset of a Hilbert space $H$ and let $S$ be a semitopological semigroup such that $R U C(S)$ has an invariant mean. Let $\mathcal{S}=\left\{T_{t}: t \in S\right\}$ be a nonexpansive semigroup on $C$ such that $\left\{T_{t} x: t \in S\right\}$ is bounded and $\cap_{s \in S} \overline{c o}\left\{T_{s t} x: t \in\right.$ $S\} \subset C$ for some $x \in C$. Then, $F(\mathcal{S}) \neq \phi$. Further, for an asymptotically invariant net $\left\{\mu_{\alpha}: \alpha \in A\right\}$ of means on $R U C(S)$, the net $\left\{T_{\mu_{\alpha}} x: \alpha \in A\right\}$ converges weakly to an element $x_{0} \in F(\mathcal{S})$.

Using Theorem 4.3, we have Theorem 4.1. By the same method, we can prove the following nonlinear ergodic theorems:

Theorem 4.4 Let $C$ be a closed convex subset of a Hilbert space $H$ and let $T$ be a oneparameter nonexpansive mapping of $C$ into itself. If $F(T)$ is nonempty, then for each $x \in C$,

$$
S_{r}(x)=(1-r) \sum_{k=0}^{\infty} r^{k} T^{k} x
$$

as $r \uparrow 1$, converges weakly to an element $y \in F(T)$.

Theorem 4.5 ([5]) Let $C$ be a closed convex subset of a Hilbert space $H$ and let $\mathcal{S}=\{S(t)$ : $t \in[0, \infty)\}$ be a nonexpansive semigroup on $C$. If $F(\mathcal{S})$ is nonempty, then for each $x \in C$,

$$
S_{\lambda}(x)=\frac{1}{\lambda} \int_{0}^{\lambda} S(t) x d t,
$$

as $\lambda \rightarrow \infty$, converges weakly to an element $y \in F(\mathcal{S})$.

Next, we state a nonlinear ergodic theorem for nonexpansive semigroups in a Banach space. Before stating it, we give a definition. A net $\left\{\mu_{\alpha}\right\}$ of continuous linear functionals on $R U C(S)$ is called strongly regular if it satisfies the following conditions: 
(i) $\sup _{\alpha}\left\|\mu_{\alpha}\right\|<+\infty$;

(ii) $\lim _{\alpha} \mu_{\alpha}(1)=1$;

(iii) $\lim _{\alpha}\left\|\mu_{\alpha}-r_{s}^{*} \mu_{\alpha}\right\|=0$ for every $s \in S$.

Theorem 4.6 ([24]) Let $S$ be a commutative semitopological semigroup and let $E$ be a unifromly convex Banach space with a Fréchet differentiable norm. Let $C$ be a nonempty closed convex subset of $E$ and let $\mathcal{S}=\left\{T_{t}: t \in S\right\}$ be a nonexpansive semigroup on $C$ such that $F(\mathcal{S})$ is nonempty. Then there exists a unique nonexpansive retraction $P$ of $C$ onto $F(\mathcal{S})$ such that $P T_{t}=T_{t} P=P$ for every $t \in S$ and $P x \in \overline{c o}\left\{T_{t} x: t \in S\right\}$ for every $x \in C$. Further, if $\left\{\mu_{\alpha}\right\}$ is a strongly regular net of continuous linear functionals on $R U C(S)$, then for each $x \in C, T_{\mu_{\alpha}} T_{t} x$ converges weakly to $P x$ uniformly in $t \in S$.

We have not known whether Theorem 4.6 would hold in the case when $S$ is noncommutative (cf. [69]). Recently, Lau, Shioji and Takahashi [36] solved the problem as follows:

Theorem 4.7 ([36]) Let $C$ be a closed convex subset of a uniformly convex Banach space $E$, let $S$ be a semitopological semigroup which $R U C(S)$ has an invariant mean, and let $\mathcal{S}=\left\{T_{t}: t \in S\right\}$ be a nonexpansive semigroup on $C$ with $F(\mathcal{S}) \neq 0$. Then there exists a nonexpansive retraction $P$ from $C$ onto $F(\mathcal{S})$ such that $P T_{t}=T_{t} P=P$ for each $t \in \mathcal{S}$ and $P x \in \overline{c o}\left\{T_{t} x: t \in S\right\}$ for each $x \in C$.

This is a generalization of Takahashi's result [64] for an amenable semigroup of nonexpansive mappings on a Hilbert space. Further they extended Rodé's result [53] to an amenable semigroup of nonexpansive mappings on a uniformly convex Banach space whose norm is Fréchet differentiable.

Theorem 4.8 ([36]) Let $E$ be a uniformly convex Banach space with a Fréchet differentiable norm and let $S$ be a semitopological semigroup. Let $C$ be a closed convex subset of $E$ and let $\mathcal{S}=\left\{T_{t}: t \in S\right\}$ be a nonexpansive semigroup on $C$ with $F(\mathcal{S}) \neq \phi$. Suppose that $R U C(S)$ has an invariant mean. Then there exists a unique nonexpansive retraction $P$ from $C$ onto $F(\mathcal{S})$ such that $P T_{t}=T_{t} P=P$ for each $t \in S$ and $P x \in \overline{c o}\left\{T_{t} x: t \in S\right\}$ for each $x \in C$. Further, if $\left\{\mu_{\alpha}\right\}$ is an asymptotically invariant net of means on $X$, then for each $x \in C,\left\{T_{\mu_{\alpha}} x\right\}$ converges weakly to $P x$.

To prove Theorem 4.8, they used Theorem 4.7 and the following lemma which has been proved in Lau, Nishiura and Takahashi [35].

Lemma 4.9 ([35]) Let $E$ be a uniformly convex Banach space with a Fréchet differentiable norm and let $S$ be a semitopological semigroup. Let $C$ be a closed convex subset of $E$ and let $\mathcal{S}=\left\{T_{t}: t \in S\right\}$ be a nonexpansive semigroup on $C$ with $F(\mathcal{S}) \neq \phi$. Then, for each $x \in C, F(\mathcal{S}) \cap \cap_{s \in S} \overline{c o}\left\{T_{t s} x: t \in S\right\}$ consists of at most one point.

The following theorem has been proved in Takahashi [66] and Lau, Nishiura and Takahashi [35] when $E$ is a Hilbert space.

Theorem 4.10 ([36]) Let $E$ be a uniformly convex Banach space with a Fréchet differentiable norm and let $S$ be a semitopological semigroup. Let $C$ be a closed convex subset of $E$ and let $\mathcal{S}=\left\{T_{t}: t \in S\right\}$ be a nonexpansive semigroup on $C$ with $F(\mathcal{S}) \neq \phi$. Suppose that for each $x \in C, F(\mathcal{S}) \cap \cap_{s \in S} \overline{c o}\left\{T_{t s} x: t \in S\right\}$ is nonempty. Then there exists a nonexpansive retraction $P$ from $C$ onto $F(\mathcal{S})$ such that $P T_{t}=T_{t} P=P$ for each $t \in S$ and $P x \in \overline{c o}\left\{T_{t} x: t \in S\right\}$ for each $x \in C$.

On the other hand, Mann [42] introduced an iteration procedure for approximating fixed points of a mapping $T$ in a Hilbert space as follows: $x_{1}=x \in C$ and

$$
x_{n+1}=\alpha_{n} x_{n}+\left(1-\alpha_{n}\right) T x_{n} \text { for } n \geq 1,
$$


where $\left\{\alpha_{n}\right\}$ is a sequence in [0,1]. Later, Reich [49] discussed this iteration procedure in a uniformly convex Banach space whose norm is Fréchet differentiable and obtained the following theorem:

Theorem 4.11 ([49]) Let $C$ be a closed convex subset of a uniformly convex Banach space $E$ with a Fréchet differentiable norm, let $T: C \rightarrow C$ be a nonexpansive mapping with a fixed point, and let $\left\{c_{n}\right\}$ be a real sequence such that $0 \leq c_{n} \leq 1$ and $\sum_{n=1}^{\infty} c_{n}\left(1-c_{n}\right)=\infty$. If $x_{1} \in C$ and

$$
x_{n+1}=c_{n} T x_{n}+\left(1-c_{n}\right) x_{n} \text { for } n \geq 1 \text {, }
$$

then $\left\{x_{n}\right\}$ converges weakly to a fixed point of $T$.

This theorem has been known for those uniformly convex Banach spaces that satisfy Opial's condition (cf. [48]). Tan and Xu [78] proved the following interesting result which generalizes the result of Reich [49].

Theorem 4.12 ([78]) Let $C$ be a closed convex subset of a uniformly convex Banach space $E$ which satisfies Opial's condition or whose norm is Fréchet differentiable and let $T: C \rightarrow$ $C$ be a nonexpansive mapping with a fixed point. Then for any initial data $x_{1}$ in $C$, the iterates $\left\{x_{n}\right\}$ defined by

$$
x_{n+1}=\alpha_{n} T\left[\beta_{n} T x_{n}+\left(1-\beta_{n}\right) x_{n}\right]+\left(1-\alpha_{n}\right) x_{n} \text { for } n \geq 1,
$$

where $\left\{\alpha_{n}\right\}$ and $\left\{\beta_{n}\right\}$ are chosen so that $\Sigma_{n=1}^{\infty} \alpha_{n}\left(1-\alpha_{n}\right)=\infty, \Sigma_{n=1}^{\infty} \beta_{n}\left(1-\alpha_{n}\right)<\infty$, $\limsup _{n \rightarrow \infty} \beta_{n}<1$, converge weakly to a fixed point of $T$.

To prove Theorem 4.12, Tan and $\mathrm{Xu}$ [78] used the following two lemmas.

Lemma 4.13 ([49],[72]) Let $C$ be a nonempty closed convex subset of a uniformly convex Banach space $E$ with a Fréchet differentiable norm and let $\left\{T_{1}, T_{2}, T_{3}, \ldots\right\}$ be a sequence of nonexpansive mappings of $C$ into $C$ such that $\cap_{n=1}^{\infty} F\left(T_{n}\right)$ is nonempty. Let $x \in C$ and put $S_{n}=T_{n} T_{n-1} \ldots T_{1}$ for $n \geq 1$. Then, the set $U \cap \cap_{n=1}^{\infty} \overline{c o}\left\{S_{m} x: m \geq n\right\}$ consists of at most one point, where $U=\cap_{n=1}^{\infty} F\left(T_{n}\right)$.

Lemma 4.14 ([54]) Let $E$ be a uniformly convex Banach space, let $\left\{t_{n}\right\}$ be a real sequence such that $0<b \leq t_{n} \leq c<1$ for $n \geq 1$ and let $a \geq 0$. Suppose that $\left\{x_{n}\right\}$ and $\left\{y_{n}\right\}$ are sequences of $E$ such that $\lim \sup _{n \rightarrow \infty}\left\|x_{n}\right\| \leq a, \limsup _{n \rightarrow \infty}\left\|y_{n}\right\| \leq a$ and $\lim _{n \rightarrow \infty} \| t_{n} x_{n}+$ $\left(1-t_{n}\right) y_{n} \|=0$. Then $\lim _{n \rightarrow \infty}\left\|x_{n}-y_{n}\right\|=\overline{0}$.

Takahashi and Kim [72] also proved the following theorem:

Theorem 4.15 ([72]) Let $E$ be a uniformly convex Banach space $E$ which satisfies Opial's condition or whose norm is Fréchet differentiable, let $C$ be a nonempty closed convex subset of $E$, and let $T: C \rightarrow C$ be a nonexpansive mapping with a fixed point. Suppose $x_{1} \in C$, and $\left\{x_{n}\right\}$ is given by

$$
x_{n+1}=\alpha_{n} T\left[\beta_{n} T x_{n}+\left(1-\beta_{n}\right) x_{n}\right]+\left(1-\alpha_{n}\right) x_{n} \text { for all } n \geq 1,
$$

where $\left\{\alpha_{n}\right\}$ and $\left\{\beta_{n}\right\}$ are chosen so that $\alpha_{n} \in[a, b]$ and $\beta_{n} \in[0, b]$ or $\alpha_{n} \in[a, 1]$ and $\beta_{n} \in[a, b]$ for some $a, b$ with $0<a \leq b<1$. Then $\left\{x_{n}\right\}$ converges weakly to a fixed point of $T$.

Motivated by Theorems 4.12 and 4.15, Suzuki and Takahashi [60] obtained the following theorem: 
Theorem 4.16 ([60]) Let $C$ be a nonempty closed convex subset of a uniformly convex Banach space $E$ which satisfies Opial's condition or whose norm is Fréchet differentiable. Let $T$ be a nonexpansive mapping from $C$ into itself with a fixed point. Suppose that $\left\{x_{n}\right\}$ is given by $x_{1} \in C$ and

$$
x_{n+1}=\alpha_{n} T\left[\beta_{n} T x_{n}+\left(1-\beta_{n}\right) x_{n}\right]+\left(1-\alpha_{n}\right) x_{n} \text { for all } n \geq 1,
$$

where $\left\{\alpha_{n}\right\}$ and $\left\{\beta_{n}\right\}$ are sequences in $[0,1]$ with $\Sigma_{n=1}^{\infty} \alpha_{n}\left(1-\alpha_{n}\right)=\infty$ and $\lim \sup _{n \rightarrow \infty} \beta_{n}<$ 1 , or $\Sigma_{n=1}^{\infty} \alpha_{n} \beta_{n}=\infty$ and $\lim \sup _{n \rightarrow \infty} \beta_{n}<1$. Then $\left\{x_{n}\right\}$ converges weakly to a fixed point of $T$.

To prove Theorem 4.16, Suzuki and Takahashi [60] used the following two lemmas. Let $I$ be an infinite subset of positive integers $N$. If $\left\{\lambda_{n}\right\}$ is a sequence of nonnegative numbers, then we denote by $\left\{\lambda_{i}: i \in I\right\}$ the subsequence of $\left\{\lambda_{n}\right\}$.

Lemma 4.17 ([60]) Let $\left\{\lambda_{n}\right\}$ and $\left\{\mu_{n}\right\}$ be sequences of nonnegative numbers such that $\Sigma_{n=1}^{\infty} \lambda_{n}=\infty$ and $\Sigma_{n=1}^{\infty} \lambda_{n} \mu_{n}<\infty$. Then for $\varepsilon>0$, there exists an infinite subset $I$ of $N$ such that $\Sigma\left\{\lambda_{j}: j \in N \backslash I\right\} \leq \varepsilon$ and the subsequence $\left\{\mu_{i}: i \in I\right\}$ of $\left\{\mu_{n}\right\}$ converges to 0 .

Lemma 4.18 ([60]) Let $\left\{\lambda_{n}\right\}$ and $\left\{\mu_{n}\right\}$ be sequences of nonnegative numbers such that $\lambda_{n+1} \leq \lambda_{n}+\mu_{n}$ for all $n \in N$. Suppose there exists a subsequence $\left\{\mu_{i}: i \in I\right\}$ of $\left\{\mu_{n}\right\}$ such that $\mu_{i} \rightarrow 0, \lambda_{i} \rightarrow \alpha$ and $\Sigma\left\{\mu_{j}: j \in N \backslash I\right\}<\infty$. Then $\lambda_{n} \rightarrow \alpha$.

Compare Theorem 4.16 with Theorem 4.12 of Tan and Xu [78]. This indicates that the assumption $\Sigma_{n=1}^{\infty} \beta_{n}\left(1-\alpha_{n}\right)<\infty$ in Theorem 4.12 is superfluous. We do not know whether the assumptions $\Sigma_{n=1}^{\infty} \alpha_{n} \beta_{n}=\infty$ and $\limsup _{n \rightarrow \infty} \beta_{n}<1$ in Theorem 4.16 are replaced by $\sum_{n=1}^{\infty} \beta_{n}\left(1-\beta_{n}\right)=\infty$ and $\liminf _{n \rightarrow \infty} \alpha_{n}>0$. We also know the following strong convergence theorem which is connected with Rhoades [51], Tan and Xu [78], and Takahashi and Kim [72].

Theorem 4.19 ([59]) Let $E$ be a strictly convex Banach space, let $C$ be a nonempty closed convex subset of $E$, and let $T: C \rightarrow C$ be a nonexpansive mapping which $T(C)$ is contained in a compact subset of $C$. Suppose $x_{1} \in C$, and $\left\{x_{n}\right\} \subset C$ is given by

$$
x_{n+1}=\alpha_{n} T\left[\beta_{n} T x_{n}+\left(1-\beta_{n}\right) x_{n}\right]+\left(1-\alpha_{n}\right) x_{n} \text { for } n \geq 1,
$$

where $\left\{\alpha_{n}\right\}$ and $\left\{\beta_{n}\right\}$ are chosen so that $\Sigma_{n=1}^{\infty} \alpha_{n}\left(1-\alpha_{n}\right)=\infty$ and $\lim \sup _{n \rightarrow \infty} \beta_{n}<1$, or $\Sigma_{n=1}^{\infty} \beta_{n}\left(1-\beta_{n}\right)=\infty$ and $\liminf _{n \rightarrow \infty} \alpha_{n}>0$. Then $\left\{x_{n}\right\}$ converges strongly to a fixed point of $T$.

Let $C$ be a closed convex subset of a Banach space $E$, and let $T, S$ be selfmaps on $C$. Then Das and Debata [14] considered the following iteration scheme: $x_{1} \in C$, and

$$
x_{n+1}=\alpha_{n} S\left[\beta_{n} T x_{n}+\left(1-\beta_{n}\right) x_{n}\right]+\left(1-\alpha_{n}\right) x_{n} \text { for } n \geq 1,
$$

where $\left\{\alpha_{n}\right\}$ and $\left\{\beta_{n}\right\}$ are real sequences in $[0,1]$. They proved a strong converence theorem concerning Roades' result [51]. Takahashi and Tamura [76] obtained the following weak convergence theorem.

Theorem 4.20 ([76]) Let $E$ be a uniformly convex Banach space $E$ which satisfies Opial's condition or whose norm is Fréchet differentiable, let $C$ be a nonempty closed convex subset of $E$, and let $S, T: C \rightarrow C$ be nonexpansive mappings such that $F(S) \cap F(T)$ is nonempty. Suppose $x_{1} \in C$, and $\left\{x_{n}\right\}$ is given by

$$
x_{n+1}=\alpha_{n} S\left[\beta_{n} T x_{n}+\left(1-\beta_{n}\right) x_{n}\right]+\left(1-\alpha_{n}\right) x_{n} \text { for } n \geq 1,
$$

where $\left\{\alpha_{n}\right\}$ and $\left\{\beta_{n}\right\}$ are chosen so that $\alpha_{n}, \beta_{n} \in[a, b]$ for some $a, b$ with $0<a \leq b<1$. Then $\left\{x_{n}\right\}$ converges weakly to a common fixed point of $S$ and $T$. 
Further, Takahashi and Tamura [76] obtained the following theorem:

Theorem 4.21 ([76]) Let $C$ be a nonempty closed convex subset of a uniformly convex Banach space $E$, and let $S, T: C \rightarrow C$ be nonexpansive mappings such that $F(S) \cap F(T)$ is nonempty. Let $P$ be the metric projection of $E$ onto $F(S) \cap F(T)$ and suppose $x_{1} \in C$, and $\left\{x_{n}\right\}$ is given by

$$
x_{n+1}=\alpha_{n} S\left[\beta_{n} T x_{n}+\left(1-\beta_{n}\right) x_{n}\right]+\left(1-\alpha_{n}\right) x_{n} \quad \text { for } n \geq 1,
$$

where $\left\{\alpha_{n}\right\}$ and $\left\{\beta_{n}\right\}$ are real sequences in $[0,1]$. Then $\left\{P x_{n}\right\}$ converges strongly to a common fixed point of $S$ and $T$.

To apply convergence theorems of Mann's type to the feasibility problem, we need to extend Theorem 4.20 to a family of finite mappings. Let $C$ be a nonempty convex subset of a Banach space $E$. Let $T_{1}, T_{2}, \ldots, T_{r}$ be finite mappings of $C$ into itself and let $\alpha_{1}, \alpha_{2}, \ldots, \alpha_{r}$ be real numbers such that $0 \leqq \alpha_{i} \leqq 1$ for every $i=1,2, \ldots, r$. Then, we define a mapping $W$ of $C$ into itself as follows:

$$
\begin{gathered}
U_{1}=\alpha_{1} T_{1}+\left(1-\alpha_{1}\right) I \\
U_{2}=\alpha_{2} T_{2} U_{1}+\left(1-\alpha_{2}\right) I, \\
\vdots \\
U_{r-1}=\alpha_{r-1} T_{r-1} U_{r-2}+\left(1-\alpha_{r-1}\right) I, \\
W=U_{r}=\alpha_{r} T_{r} U_{r-1}+\left(1-\alpha_{r}\right) I .
\end{gathered}
$$

Such a $W$ is called the $W$-mapping generated by $T_{1}, T_{2}, \ldots, T_{r}$ and $\alpha_{1}, \alpha_{2}, \ldots, \alpha_{r}$.

Theorem 4.22 ([74]) Let $E$ be a uniformly convex Banach space $E$ which satisfies Opial's condition or whose norm is Fréchet differentiable, let $C$ be a nonempty closed convex subset of $E$, and let $\left\{T_{1}, T_{2}, \ldots, T_{r}\right\}$ be finite nonexpansive mappings of $C$ into itself such that $\cap_{n=1}^{r} F\left(T_{n}\right)$ is nonempty. Let $a, b$ be real numbers with $0<a \leq b<1$ and suppose $x_{1} \in C$, and $\left\{x_{n}\right\}$ is given by

$$
x_{n+1}=W_{n} x_{n} \text { for } n \geq 1 \text {, }
$$

where $W_{n}$ are $W$-mappings generated by $T_{1}, T_{2}, \ldots, T_{r}$ and $\alpha_{n 1}, \alpha_{n 2}, \cdots, \alpha_{n r} \in[a, b]$. Then $\left\{x_{n}\right\}$ converges weakly to a common fixed point of $T_{1}, T_{2}, \ldots, T_{r}$.

We will finally show a weak convergence theorem of Mann's type for a nonexpansive semigroup in a Banach space.

Theorem 4.23 ([1]) Let $E$ be a uniformly convex Banach space $E$ with a Fréchet differentiable norm. Let $C$ be a nonempty closed convex subset of $E$ and let $\mathcal{S}=\left\{T_{t}: t \in S\right\}$ be a nonexpansive semigroup on $C$ such that $F(\mathcal{S}) \neq \phi$. Let $\left\{\mu_{n}\right\}$ be a sequence of means on $R C U(S)$ such that $\left\|\mu_{n}-\ell_{s}^{*} \mu_{n}\right\|=0$ for every $s \in S$. Suppose $x_{1}=x \in C$ and $\left\{x_{n}\right\}$ is given by

$$
x_{n+1}=\alpha_{n} x_{n}+\left(1-\alpha_{n}\right) T_{\mu_{n}} x_{n} \quad \text { for every } n \geq 1,
$$

where $\left\{\alpha_{n}\right\}$ is a sequence in $[0,1]$. If $\left\{\alpha_{n}\right\}$ is chosen so that $\alpha_{n} \in[0, a]$ for some a with $0<a<1$, then $\left\{x_{n}\right\}$ converges weakly to an element $x_{0} \in F(\mathcal{S})$.

Using Theorem 4.23, we can prove a weak convergence theorem of Mann's type for a one-parameter nonexpansive semigroup. 
Theorem 4.24 Let $E$ be a uniformly convex Banach space $E$ with a Fréchet differentiable norm and let $C$ be a closed convex subset of $E$. Let $\mathcal{S}=\{\mathcal{S}(t): t \in[0, \infty)\}$ be a oneparameter nonexpansive semigroup on $C$ such that $F(\mathcal{S}) \neq \phi$. Suppose $x_{1}=x \in C$ and $\left\{x_{n}\right\}$ is given by

$$
x_{n+1}=\alpha_{n} x_{n}+\left(1-\alpha_{n}\right) \frac{1}{s_{n}} \int_{0}^{s_{n}} S(t) x_{n} d t \quad \text { for every } n \geqq 1,
$$

where $s_{n} \rightarrow \infty$ as $n \rightarrow \infty$ and $\left\{\alpha_{n}\right\}$ is a sequence in $[0,1]$. If $\left\{\alpha_{n}\right\}$ is chosen so that $\alpha_{n} \in[0, a]$ for some a with $0<a<1$, then $\left\{x_{n}\right\}$ converges weakly to a common fixed point $z \in F(\mathcal{S})$.

\section{Strong Convergence Theorems}

In this section,we discuss strong convergence theorems for nonexpansive mappings. Let $C$ be a nonempty closed convex subset of a real Hilbert space $H$. In 1967, Browder [10] obtained the following strong convergence theorem: For a given $u \in C$ and each $n \in N$, define a contraction $T_{n}: C \rightarrow C$ by

$$
T_{n} x=\left(1-\frac{1}{n}\right) T x+\frac{1}{n} u \text { for all } x \in C,
$$

where $T$ is a nonexpansive mapping of $C$ into itself. Then, there exists a unique fixed point $x_{n}$ of $T_{n}$ in $C$ such that

$$
x_{n}=\left(1-\frac{1}{n}\right) T x_{n}+\frac{1}{n} u .
$$

Further if the set $F(T)$ of fixed points of $T$ is nonempty, then $\left\{x_{n}\right\}$ converges strongly as $n \rightarrow \infty$ to a fixed point of $T$. After Browder's result, such a problem has been investigated by several authors. In particular, Reich [50] and Takahashi and Ueda [77] also extended Browder's result to strong convergence theorems for resolvents of accretive operators in a Banach space. Before stating them, we give two definitions. A closed convex subset $C$ of a Banach space $E$ is said to have the fixed point property for nonexpansive mappings if every nonexpansive mapping of $C$ into itself has a fixed point in every nonempty bounded closed convex subset of $C$ such that $T$ leaves invariant. Let $A$ be an accretive operator in a Banach space $E$. Then $A$ is said to satisfy the range condition if $\overline{D(A)} \subset R(I+r A)$ for every $r>0$. Now we can prove the first strong convergence theorem for resolvents of accretive operators.

Theorem 5.1 ([77]) Let $E$ be a reflexive Banach space with a uniformly Gâteaux differentiable norm and let $A \subset E \times E$ be an accretive operator that satisfies the range condition. Let $C$ be a closed convex subset of $E$ such that

$$
\overline{D(A)} \subset C \subset \bigcap_{r>0} R(I+r A)
$$

and every weakly compact convex subset of $C$ has the fixed point property for nonexpansive mappings. If $0 \in R(A)$, then for each $x$ in $C, \lim _{t \rightarrow \infty} J_{t} x$ exists and belongs to $A^{-1} 0$.

As direct consequences of Theorem 5.1, we obtain the following two results.

Theorem 5.2 ([50]) Let $E$ be a uniformly convex and uniformly smooth Banach space, and let $A \subset E \times E$ be $m$-accretive. If $0 \in R(A)$, then for each $x \in E, \lim _{t \rightarrow \infty} J_{t} x$ exists and belongs to $A^{-1} 0$. 
Theorem 5.3 ([50]) Let $E$ be a reflexive Banach space with a uniformly Gâteaux differentiable norm, let $A \subset E \times E$ be an accretive operator that satisfies the range condition. Suppose that every weakly compact convex subset of $E$ has the fixed point property for nonexpansive mappings. If $A^{-1} 0 \neq \phi$ and $\overline{D(A)}$ is convex, then for each $x \in \overline{D(A)}, \lim _{t \rightarrow \infty} J_{t} x$ exists and belongs to $A^{-1} 0$.

We also know the following theorem:

Theorem 5.4 ([67]) Let $C$ be a closed convex subset of a Banach space $E$ and let $T$ be a nonexpansive mapping of $C$ into itself. Then the following hold:

(i) If $A=I-T$, then $A$ is accretive;

(ii) $C=D(A) \subset \bigcap_{r>0} R(I+r A)$.

Theorem 5.3 generalizes Browder's strong convergence theorem. In fact, from

$$
x_{n}=\left(1-\frac{1}{n}\right) T x_{n}+\frac{1}{n} u,
$$

we have

$$
x_{n}+(n-1)(I-T) x_{n}=u .
$$

Putting $A=I-T$, we have from Thorem 5.4 that $A$ is accretive and satisfies the range condition. Since $J_{n-1} u=x_{n}$ from $(* * *)$, we have, by Theorem 5.3 ,

$$
\lim _{n \rightarrow \infty} J_{n} u=\lim _{n \rightarrow \infty} x_{n+1} \in(I-T)^{-1} 0=F(T) \text {. }
$$

Recently, Wittmann [80] dealt with the following iterative process in a Hilbert space: $x_{1}=x \in C$ and

$$
x_{n+1}=\alpha_{n} x+\left(1-\alpha_{n}\right) T x_{n} \text { for } n \geq 1,
$$

where $\left\{\alpha_{n}\right\}$ is a sequence in $[0,1]$; see originally Halpern [23]. The following theorem was proved by Wittmann.

Theorem 5.5 ([80]) Let $H$ be a Hilbert space. Let $C$ be a nonempty closed convex subset of $H$. Let $T$ be a nonexpansive mapping of $C$ into itself such that $F(T) \neq \emptyset$. Let $\left\{\beta_{n}\right\}$ be a sequence of real numbers such that $0 \leq \beta_{n} \leq 1, \lim _{n \rightarrow \infty} \beta_{n}=0, \sum_{n=1}^{\infty}\left|\beta_{n+1}-\beta_{n}\right|<\infty$ and $\sum_{n=1}^{\infty} \beta_{n}=\infty$. Suppose that $\left\{x_{n}\right\}$ is given by $x_{1}=x \in C$ and

$$
x_{n+1}=\beta_{n} x+\left(1-\beta_{n}\right) T x_{n} \text { for } n \geq 1 .
$$

Then, $\left\{x_{n}\right\}$ converges strongly to $P x \in F(T)$, where $P$ is the metric projection from $C$ onto $F(T)$.

Shioji and Takahashi [56] extended Wittmann's theorem to a Banach space by using Theorem 5.1 as follows:

Theorem 5.6 ([56]) Let $E$ be a uniformly convex Banach space with a uniformly Gâteaux differentiable norm. Let $C$ be a nonempty closed convex subset of $E$. Let $T$ be a nonexpansive mapping of $C$ into itself such that $F(T) \neq \emptyset$. Let $\left\{\beta_{n}\right\}$ be a sequence of real numbers such that $0 \leq \beta_{n} \leq 1, \lim _{n \rightarrow \infty} \beta_{n}=0, \sum_{n=1}^{\infty}\left|\beta_{n+1}-\beta_{n}\right|<\infty$ and $\sum_{n=1}^{\infty} \beta_{n}=\infty$. Suppose that $\left\{x_{n}\right\}$ is given by $x_{1}=x \in C$ and

$$
x_{n+1}=\beta_{n} x+\left(1-\beta_{n}\right) T x_{n} \text { for } n \geq 1 \text {. }
$$

Then, $\left\{x_{n}\right\}$ converges strongly to $P x \in F(T)$, where $P$ is a unique sunny nonexpansive retraction from $C$ onto $F(T)$. 
Kamimura and Takahashi [30] also obtained the following result by using Theorem 5.1, which is connected with the proximal point algorithm.

Theorem 5.7 ([30]) Let $E$ be a uniformly convex Banach space with a uniformly Gâteaux differentiable norm and let $A \subset E \times E$ be an m-accretive operator. Let $x \in E$ and let $\left\{x_{n}\right\}$ be a sequence defined by $x_{1}=x$ and

$$
x_{n+1}=\alpha_{n} x+\left(1-\alpha_{n}\right) J_{r_{n}} x_{n} \quad \text { for } n \geqq 1,
$$

where $\left\{\alpha_{n}\right\} \subset[0,1]$ and $\left\{r_{n}\right\} \subset(0, \infty)$ satisfy $\lim _{n \rightarrow \infty} \alpha_{n}=0, \sum_{n=1}^{\infty} \alpha_{n}=\infty$ and $\lim _{n \rightarrow \infty} r_{n}=$ $\infty$. If $A^{-1} 0 \neq \phi$, then $\left\{x_{n}\right\}$ converges strongly to an element $P x \in A^{-1} 0$, where $P$ is a unique sunny nonexpansive retraction of $E$ onto $A^{-1} 0$.

Atsushiba and Takahashi [3] proved a strong convergence theorem for finite nonexpansive mappings which is connected with the feasibility problem.

Theorem 5.8 Let $E$ be a uniformly convex Banach space with a uniformly Gâteaux differentiable norm. Let $C$ be a nonempty closed convex subset of $E$, let $\alpha_{n 1}, \alpha_{n 2}, \ldots, \alpha_{n r}$ be real numbers such that $0<\alpha_{n i}<1$ for every $i=1,2, \ldots, r-1$ and $n=1,2, \ldots, 0<\alpha_{n r} \leqq 1$ for every $n=1,2, \cdots$ and let $T_{1}, T_{2}, \cdots, T_{r}$ be finite nonexpansive mappings of $C$ into itself such that $\cap_{i=1}^{r} F\left(T_{i}\right) \neq \emptyset$. Let $W_{n}(n=1,2, \cdots)$ be the $W$-mappings of $C$ into itself generated by $T_{1}, T_{2}, \cdots, T_{r}$ and $\alpha_{n 1}, \alpha_{n 2}, \cdots, \alpha_{n r}$. Let $\left\{\beta_{n}\right\}$ be a sequence of real numbers such that $0 \leqq \beta_{n} \leqq 1$ for every $n=1,2, \cdots, \lim _{n \rightarrow \infty} \beta_{n}=0, \sum_{n=1}^{\infty}\left|\beta_{n+1}-\beta_{n}\right|<\infty$ and $\sum_{n=1}^{\infty} \beta_{n}=\infty$. Suppose that $\sum_{n=1}^{\infty}\left|\alpha_{n+1 i}-\alpha_{n i}\right|<\infty$ for every $i=1,2, \cdots, r$ and $\left\{x_{n}\right\}$ is given by $x_{1}=x \in C$ and

$$
x_{n+1}=\beta_{n} x+\left(1-\beta_{n}\right) W_{n} x_{n}
$$

for every $n=1,2, \cdots$. Then, $\left\{x_{n}\right\}$ converges strongly to $P x \in \cap_{n=1}^{\infty} F\left(W_{n}\right)=\cap_{i=1}^{r} F\left(T_{i}\right)$, where $P$ is a unique sunny nonexpansive retraction from $C$ onto $\cap_{i=1}^{r} F\left(T_{i}\right)$.

We will finally show a strong convergence theorem [58] for a nonexpansive semigroup in a Banach space without compactness.

Theorem 5.9 ([58]) Let $E$ be a uniformly convex Banach space $E$ with a uniformly Gâteaux differentiable norm. Let $C$ be a nonempty closed convex subset of $E$ and let $\mathcal{S}=\left\{T_{t}: t \in S\right\}$ be a nonexpansive semigroup on $C$ such that $F(\mathcal{S}) \neq \phi$. Let $\left\{\mu_{n}\right\}$ be a sequence of means on $R U C(S)$ such that $\left\|\mu_{n}-\ell_{s}^{*} \mu_{n}\right\|=0$ for every $s \in S$. Suppose $x, y_{1} \in C$ and $\left\{y_{n}\right\}$ is given by

$$
y_{n+1}=\beta_{n} x+\left(1-\beta_{n}\right) T_{\mu_{n}} y_{n} \quad \text { for every } n \geq 1,
$$

where $\left\{\beta_{n}\right\}$ is in $[0,1]$. If $\left\{\beta_{n}\right\}$ is chosen so that $\lim _{n \rightarrow \infty} \beta_{n}=0$ and $\Sigma_{n=1}^{\infty} \beta_{n}=\infty$, then $\left\{y_{n}\right\}$ converges strongly to the element of $F(\mathcal{S})$ which is nearest to $x$ in $F(\mathcal{S})$.

Using Theorem 5.9, we can prove a strong convergence theorem for a one-parameter nonexpansive semigroup.

Theorem 5.10 Let $E$ be a uniformly convex Banach space $E$ with a uniformly Gâteaux differentiable norm. Let $C$ be a nonempty closed convex subset of $E$ and let $\mathcal{S}=\{\mathcal{S}(t): t \geqq$ $0\}$ be a one-parameter nonexpansive semigroup on $C$ such that $F(\mathcal{S}) \neq \emptyset$. Suppose $x, y_{1} \in \bar{C}$ and $\left\{y_{n}\right\}$ is given by

$$
y_{n+1}=\beta_{n} x+\left(1-\beta_{n}\right) \frac{1}{\lambda_{n}} \int_{0}^{\lambda_{n}} S(t) y_{n} d t \quad \text { for every } n \geqq 1,
$$

where $\left\{\beta_{n}\right\}$ is a sequence in $[0,1]$. If $\left\{\beta_{n}\right\}$ and $\left\{\lambda_{n}\right\}$ are chosen so that $\lim _{n \rightarrow \infty} \beta_{n}=0$, $\sum_{n=1}^{\infty} \beta_{n}=\infty$ and $\lambda_{n} \rightarrow \infty$, then $\left\{y_{n}\right\}$ converges strongly to the element of $F(\mathcal{S})$ which is nearest to $x$ in $F(\mathcal{S})$. 


\section{Applications}

In this section, we first deal with convergence theorems which are connected with the feasibility problem; for the feasibility problem, see Section 1. Using a nonlinear ergodic theorem, Grombez [13] considerd the feasibility problem in a Hilbert space setting. Let $H$ be a Hilbert space, let $C_{1}, C_{2}, \ldots, C_{r}$ be nonempty closed convex subsets of $H$ and let $I$ be the identity operator on $H$. Then the feasibility problem in a Hilbert space setting may be stated as follows: The original (unknown) image $z$ is known a priori to belong to the intersection $C_{0}$ of $r$ well-defined sets $C_{1}, C_{2}, \ldots, C_{r}$ in a Hilbert space; given only the metric projections $P_{i}$ of $H$ onto $C_{i}(i=1,2, \ldots, r)$, recover $z$ by an iterative scheme. Crombez [13] proved the following: Let $T=\alpha_{0} I+\sum_{i=0}^{r} \alpha_{i} T_{i}$ with $T_{i}=I+\lambda_{i}\left(P_{i}-I\right)$ for all $i, 0<\lambda_{i}<2$, $\alpha_{i} \geqq 0$ for $i=0,1,2, \ldots, r, \sum_{i=0}^{r} \alpha_{i}=1$ where each $P_{i}$ is the metric projection of $H$ onto $C_{i}$ and $C_{0}=\cap_{i=1}^{r} C_{i}$ is nonempty. Then starting from an arbitrary element $x$ of $H$, the sequence $\left\{T^{n} x\right\}$ converges weakly to an element of $C_{0}$. Later, Kitahara and Takahashi [32] and Takahashi and Tamura [75] dealt with the feasibility problem by convex combinations of sunny nonexpansive retractions in uniformly convex Banach spaces.

Theorem 6.1 ([75]) Let $E$ be a uniformly convex Banach space with a Fréchet differentiable norm and let $C$ be a nonempty closed convex subset of $E$. Let $C_{1}, C_{2}, \ldots, C_{r}$ be nonexpansive retracts of $C$ such that $\cap_{i=1}^{r} C_{i} \neq \neq$. Let $T$ be a mapping on $C$ given by $T=\sum_{i=1}^{r} \alpha_{i} T_{i}, 0<\alpha_{i}<1, \sum_{i=1}^{r} \alpha_{i}=1$, such that for each $i, T_{i}=\left(1-\lambda_{i}\right) I+\lambda_{i} P_{i}$, $0<\lambda_{i}<1$, where $P_{i}$ is a nonexpansive retraction of $C$ onto $C_{i}$. Then, $F(T)=\cap_{i=1}^{r} C_{i}$ and further, for each $x \in C,\left\{T^{n} x\right\}$ converges weakly to an element of $\cap_{i=1}^{r} C_{i}$.

Takahashi and Shimoji [74] solved the feasibility problem by using the convergence theorem of Mann's type (Theorem 4.22).

Theorem 6.2 ([74]) Let $E$ be a unifomly convex Banach space with a Fréchet differentiable norm. Let $C_{1}, C_{2}, \ldots, C_{r}$ be nonexpansive retracts of $C$ such that $\cap_{i=1}^{r} C_{i} \neq \phi$. Let $W$ be the $W$-mapping of $C$ into itself generated by $P_{1}, P_{2}, \ldots, P_{r}$ and $\alpha_{1}, \alpha_{2}, \ldots, \alpha_{r}$ where $P_{i}$ is a nonexpansive retraction of $C$ onto $C_{i}$ and $0<\alpha_{i}<1$ for every $i=1,2, \ldots, r$. Then for each $x \in C,\left\{W^{n} x\right\}$ converges weakly to an element of $\cap_{i=1}^{r} C_{i}$.

When $\cap_{i=1}^{r} C_{i}$ is empty in Theorem 6.2 , we have the following two theorems.

Theorem 6.3 ([74]) Let $E$ be a reflexive Banach space and let $C$ be a nonempty closed convex subset of $E$ which has normal structure. Let $C_{1}, C_{2}, \ldots, C_{r}$ be nonempty bounded nonexpansive retracts of $C$. Let $W$ be the $W$-mapping generated by $P_{1}, P_{2}, \ldots, P_{r}$ and $\alpha_{1}, \alpha_{2}, \ldots, \alpha_{r}$, where $0<\alpha_{1}, \ldots, \alpha_{r}<1$ and $P_{i}$ is a nonexpansive retraction of $C$ onto $C_{i}$. Then $F(W)$ is nonempty. Further, assume that $E$ is strictly convex and $\cap_{i=1}^{r} C_{i}=\emptyset$. Then $F(W) \cap C_{i}=\emptyset$ for some $i$.

Let $C$ and $D$ be nonempty convex subsets of a Banach space $E$. Then we denote by $i_{C} D$ the set of $z \in D$ such that for any $x \in C$, there exists $\lambda \in(0,1)$ with $\lambda x+(1-\lambda) z \in D$ and by $\partial_{C} D$ the set of $z \in D$ such that there exists $x \in C$ with $\lambda x+(1-\lambda) z \notin D$ for all $\lambda \in(0,1)$.

Theorem 6.4 ([74]) Let $E$ be a strictly convex and reflexive Banach space and let $C$ be a nonempty closed convex subset of $E$ which has normal structure. Let $C_{1}, C_{2}, \ldots, C_{r}$ be nonempty bounded sunny nonexpansive retracts of $C$ such that for each $i$, an element of $\partial_{C} C_{i}$ is an extreme point of $C_{i}$. Let $W$ be the $W$-mapping generated $P_{1}, P_{2}, \ldots, P_{r}$ and $\alpha_{1}, \alpha_{2}, \ldots, \alpha_{r}$, where $0<\alpha_{1}, \ldots, \alpha_{r}<1$ and $P_{i}$ is a sunny nonexpansive retraction of $C$ onto $C_{i}$. If $\cap_{i=1}^{r} C_{i}$ is empty, then $F(W)$ consists of one point.

Atsushiba and Takahashi [3] proved the following strong convergence theorem. 
Theorem 6.5 ([3]) Let $E$ be a uniformly convex Banach space with a uniformly Gâteaux differentiable norm, let $C$ be a nonempty closed convex subset of $E$ and let $C_{1}, C_{2}, \ldots, C_{r}$ be nonexpansive retracts of $C$ such that $\cap_{i=1}^{r} C_{i} \neq \emptyset$. Let $W$ be the $W$-mapping of $C$ into itself generated by $P_{1}, P_{2}, \ldots, P_{r}$ and $\alpha_{1}, \alpha_{2}, \ldots, \alpha_{r}$, where $P_{i}(i=1,2, \ldots, r)$ is a nonexpansive retraction from $C$ onto $C_{i}$ and $0<\alpha_{i}<1$ for every $i=1,2, \ldots, r-1$ and $0<\alpha_{r} \leq 1$. Let $\left\{\beta_{n}\right\}$ be a sequence of real numbers such that $0 \leq \beta_{n} \leq 1$ for every $n=1,2, \ldots, \lim _{n \rightarrow \infty} \beta_{n}=$ $0, \sum_{n=1}^{\infty}\left|\beta_{n+1}-\beta_{n}\right|<\infty$ and $\sum_{n=1}^{\infty} \beta_{n}=\infty$. If $\left\{y_{n}\right\}$ is given by $y_{1}=x \in C$ and

$$
y_{n+1}=\beta_{n} x+\left(1-\beta_{n}\right) W y_{n}
$$

for every $n=1,2, \ldots$, then $\left\{y_{n}\right\}$ converges strongly to $P x \in F(W)=\cap_{i=1}^{r} C_{i}$, where $P$ is a unique sunny nonexpansive retraction from $C$ onto $F(W)=\cap_{i=1}^{r} C_{i}$.

Using Theorems 6.2 and 6.5 , we consider the problem of finding a common fixed point for a finite commuting family of nonexpansive mappings.

Theorem 6.6 ([74]) Let $E$ be a uniformly convex Banach with a Féchet differentiable norm and let $C$ be a nonempty closed convex subset of $E$. Let $\left\{S_{1}, S_{2}, \ldots, S_{r}\right\}$ be a commuting finite family of nonexpansive mappings on $C$ with $F\left(S_{i}\right) \neq \emptyset, i=1,2, \ldots, r$. Let $W$ be the $W$-mapping generated by $P_{1}, P_{2}, \ldots, P_{r}$ and $\alpha_{1}, \alpha_{2}, \ldots, \alpha_{r}$ where for each $i, 0<\alpha_{i}<1$ and $P_{i}$ is a nonexpansive retraction of $C$ onto $F\left(S_{i}\right)$. Then, $F(W)=\cap_{i=1}^{r} F\left(S_{i}\right)$ is nonempty. Further, for each $x \in C,\left\{W^{n} x\right\}$ converges weakly to an element of $\cap_{i=1}^{r} F\left(S_{i}\right)$.

Theorem 6.7 ([3]) Let $E$ be a uniformly convex Banach with a uniformly Gâteaux differentiable norm and let $C$ be a closed convex subset of $E$. Let $\left\{S_{1}, S_{2}, \ldots, S_{r}\right\}$ be a commuting finite family of nonexpansive mappings of $C$ into itself with $F\left(S_{i}\right) \neq \phi, i=1,2, \ldots, r$. Let $W$ be the $W$-mapping generated by $P_{1}, P_{2}, \ldots, P_{r}$ and $\alpha_{1}, \alpha_{2}, \ldots, \alpha_{r}$, where $0<\alpha_{i}<1$ for every $i=1,2, \ldots, r-1,0<\alpha_{r} \leqq 1$ and $P_{i}$ is a unique sunny nonexpansive retraction from $C$ onto $F\left(S_{i}\right)$ for every $i=1,2, \ldots, r$. Then, $F(W)=\cap_{i=1}^{r} F\left(S_{i}\right) \neq \phi$. Let $\left\{\beta_{n}\right\}$ be a sequence of real numbers such that $0 \leqq \beta_{n} \leqq 1$ for every $n=1,2, \ldots, \lim _{n \rightarrow \infty} \beta_{n}=$ $0, \sum_{n=1}^{\infty}\left|\beta_{n+1}-\beta_{n}\right|<\infty$ and $\sum_{n=1}^{\infty} \beta_{n}=\infty$. Suppose $y_{1}=x \in C$ and $\left\{y_{n}\right\}$ is given by

$$
y_{n+1}=\beta_{n} x+\left(1-\beta_{n}\right) W y_{n}
$$

for every $n=1,2, \ldots$ Then, $\left\{y_{n}\right\}$ converges strongly to $P x \in F(W)$, where $P$ is a unique sunny nonexpansive retraction from $C$ onto $F(W)=\cap_{i=1}^{r} F\left(S_{i}\right)$.

Finally, we consider two proximal point algorithms for sloving $(*)$ in Section 1, with parameters $\left\{r_{n}\right\}$, starting at an initial point $x_{1}$ in a Hilbert space $H$. As a direct consequence of Theorem 5.7, we obtain the following result.

Theorem 6.8 Let $H$ be a Hilbert space and $A \subset H \times H$ be an m-accretive operator. Let $x \in H$ and let $\left\{x_{n}\right\}$ be a sequence defined by $x_{1}=x$ and

$$
x_{n+1}=\alpha_{n} x+\left(1-\alpha_{n}\right) J_{r_{n}} x_{n} \quad \text { for } n \geqq 1,
$$

where $\left\{\alpha_{n}\right\} \subset[0,1]$ and $\left\{r_{n}\right\} \subset(0, \infty)$ satisfy $\lim _{n \rightarrow \infty} \alpha_{n}=0, \sum_{n=1}^{\infty} \alpha_{n}=\infty$ and $\lim _{n \rightarrow \infty} r_{n}=$ $\infty$. If $A^{-1} 0 \neq \phi$, then $\left\{x_{n}\right\}$ converges strongly to $P x \in A^{-1} 0$, where $P$ is the metric projection of $H$ onto $A^{-1} 0$.

Using Theorem 6.8, we obtain the following theorem. 
Theorem 6.9 ([29]) Let $H$ be a Hilbert space and let $f: H \rightarrow(-\infty, \infty]$ be a lower semicontinuous proper convex function. Let $x \in H$ and let $\left\{x_{n}\right\}$ be a sequence defined by $x_{1}=x$ and

$$
\begin{gathered}
x_{n+1}=\alpha_{n} x+\left(1-\alpha_{n}\right) J_{r_{n}} x_{n} \quad \text { for } n \geqq 1, \\
J_{r_{n}} x_{n}=\arg \min \left\{f(z)+\frac{1}{2 r_{n}}\left\|z-x_{n}\right\|^{2}: z \in H\right\},
\end{gathered}
$$

where $\left\{\alpha_{n}\right\} \subset[0,1]$ and $\left\{r_{n}\right\} \subset(0, \infty)$ satisfy $\lim _{n \rightarrow \infty} \alpha_{n}=0, \sum_{n=1}^{\infty} \alpha_{n}=\infty$ and $\lim _{n \rightarrow \infty} r_{n}=$ $\infty$. If $(\partial f)^{-1} 0 \neq \phi$, then $\left\{x_{n}\right\}$ converges strongly to $v \in H$, which is the minimizer of $f$ nearest to $x$. Further

$$
f\left(x_{n+1}\right)-f(v) \leqq \alpha_{n}(f(x)-f(v))+\frac{1-\alpha_{n}}{r_{n}}\left\|J_{r_{n}} x_{n}-v\right\|\left\|J_{r_{n}} x_{n}-x_{n}\right\| .
$$

The following is the proximal point algorithm by the Mann iteration proceduce.

Theorem 6.10 ([29]) Let $H$ be a Hilbert space and let $f: H \rightarrow(-\infty, \infty]$ be a lower semicontinuous proper convex function. Let $x \in H$ and let $\left\{x_{n}\right\}$ be a sequence defined by $x_{1}=x$ and

$$
\begin{gathered}
x_{n+1}=\alpha_{n} x_{n}+\left(1-\alpha_{n}\right) J_{r_{n}} x_{n} \quad \text { for } n \geqq 1, \\
J_{r_{n}} x_{n}=\arg \min \left\{f(z)+\frac{1}{2 r_{n}}\left\|z-x_{n}\right\|^{2}: z \in H\right\},
\end{gathered}
$$

where $\left\{\alpha_{n}\right\} \subset[0,1]$ and $\left\{r_{n}\right\} \subset(0, \infty)$ satisfy $\alpha_{n} \in[0, k]$ for some $k$ with $0<k<1$ and $\lim _{n \rightarrow \infty} r_{n}=\infty$. If $(\partial f)^{-1} 0 \neq \phi$, then $\left\{x_{n}\right\}$ converges weakly to $v \in H$, which is a minimizer of $f$. Further

$$
f\left(x_{n+1}\right)-f(v) \leqq \alpha_{n}\left(f\left(x_{n}\right)-f(v)\right)+\frac{1-\alpha_{n}}{r_{n}}\left\|J_{r_{n}} x_{n}-v\right\|\left\|J_{r_{n}} x_{n}-x_{n}\right\| .
$$

Acknowledgments. The author would like to thank two anonymous referees for their valuable comments.

\section{References}

[1] S. Atsushiba, N. Shioji and W. Takahashi: Approximating common fixed points by the Mann iteration process in Banach spaces. J. Nonlinear Convex Anal., to appear.

[2] S. Atsushiba and W. Takahashi: Approximating common fixed points of nonexpansive semigroups by the Mann iteration process. Ann. Univ. Mariae Curie-Sklodowska, 51 (1997) $1-16$.

[3] S. Atushiba and W. Takahashi: Strong convergence theorems for a finite family of nonexpansive mappings and applications. Indian J. Math., to appear.

[4] J. B. Baillon: Un théorème de type ergodic pour les contraction non linéaires dans un espace de Hilbert. C. R. Acad. Sci. Paris, 280 (1975) 1511-1514.

[5] J. B. Baillon: Quelques properiétès de convergence asymptotique pour les semigroupes de contractions impaìres. C. R. Acad. Sci. Paris, 283 (1976) 75-78.

[6] J. B. Baillon and R. Schöneberg: Asymptotic normal structure and fixed points of nonexpansive mappings. Proc. Amer. Math. Soc., 81 (1981) 257-264.

[7] H.Brèzis: Opérateurs Maximaux Monotones. Mathematics Studies No.5( North-Holland, Amsterdam, 1973). 
[8] F. E. Browder: Fixed point theorems for noncompact mappings in Hilbert space. Proc. Nat. Sci. USA, 43 (1965) 1272-1276.

[9] F. E. Browder: Nonexpansive nonlinear operators in a Banach space. Proc. Nat. Acad. Sci. USA, 54 (1965) 1041-1044.

[10] F. E. Browder: Convergence of approximants to fixed points of nonexpansive nonlinear mappings in Banach space. Archs. Ratio. Anal., 24 (1967) 82-90.

[11] R. E. Bruck: A common fixed point theorem for a commuting family of nonexpansive mappings. Pacific J. Math., 53 (1974) 59-71.

[12] R. E. Bruck: A simple proof of the mean ergodic theorem for nonlinear contractions in Banach spaces. Israel J. Math., 32 (1979) 107-116.

[13] G. Crombez: Image recovery by convex combinations of projections. J. Math. Anal. Appl., 155 (1991) 413-419.

[14] G. Das and J. P. Debata: Fixed points of quasinonexpansive mappings. Indian J. Pure Appl. Math., 17 (1986) 1263-1269.

[15] M. M. Day: Amenable semigroups. Illinois J. Math., 1 (1957) 509-544.

[16] E. DeMarr: Common fixed points for commuting contraction mappings. Pacific J. Math., 13 (1963) 1139-1141.

[17] J. Diestel: Geometry of Banach spaces, Selected Topics. Lecture Notes in Mathematics 485 (Springer, Berlin, 1975).

[18] M. Edelstein and R. C. O'Brien: Nonexpansive mappings, asymptotic regularity and successive approximations. J. London Math. Soc., 17 (1978) 547-554.

[19] K. Fan: Existence theorems and extreme solutions for inequalities concerning convex functions or linear transformations. Math. Z., 68 (1957) 205-217.

[20] D Göhde: Zum prinzip der kontraktiven abbildung. Math. Nach., 30 (1965) 251-258.

[21] C. W. Groetsch: A note on segmenting Mann iterates. J. Math. Anal. Appl., 40 (1972) 369-372.

[22] O. Güler: On the convergence of the proximal point algorithm for convex minimization. SIAM J. Control Optim., 29 (1991) 403-419.

[23] B. Halpern: Fixed points of nonexpanding maps. Bull. Amer. Math. Soc., 73 (1967) 957-961.

[24] N. Hirano, K. Kido and W. Takahashi: Nonexpansive retractions and nonlinear ergodic theorems in Banach spaces. Nonlinear Analysis, 12 (1988) 1269-1281.

[25] S. Ishikawa: Fixed points and iteration of a nonexpansive mapping in a Banach space. Proc. Amer. Math. Soc., 59 (1976) 65-71.

[26] O. Kada, A. T. Lau and W. Takahashi: Asymptotically invariant net and fixed point set for semigroup of nonexpansive mappings. Nonlinear Analysis, 29 (1997) 539-550.

[27] O. Kada and W. Takahashi: Nonlinear ergodic theorems of almost nonexpansive curves for commutative semigroups. Topol. Methods Nonlinear Anal., 5 (1995) 305-324.

[28] O. Kada and W. Takahashi: Strong convergence and nonlinear ergodic theorems for commutative semigroups of nonexpansive mappings. Nonlinear Analysis, 28 (1997) 495-511.

[29] S. Kamimura and W. Takahashi: Approximating solutions of maximal monotone operators in Hilbert spaces. J. Approximation Theory, to appear.

[30] S. Kamimura and W. Takahashi: Weak and strong convergence to solutions of accretive operators and applications. Set-Valued Analysis, to appear.

[31] W. A. Kirk: A fixed point theorem for mappings which do not increase distances. Amer. Math. Monthly, 72 (1965) 1004-1006.

[32] S. Kitahara and W. Takahashi: Image recovery by convex combinations of sunny nonexpansive retractions. Topol. Methods Nonlinear Analysis, 2 (1993) 333-342. 
[33] A. T. Lau: Invariant means on almost periodic functions and fixed point properties. Rocky Mountain J. Math., 3 (1973) 69-76.

[34] A. T. Lau: Amenability and fixed point property for semigroup of non-expansive mappings. In M. A. Théra and J. B. Baillon (eds.): Fixed Point Theory and Applications, Pitman Research Notes in Mathematics Series \#252 (1991) 303-313.

[35] A. T. Lau, K. Nishiura and W. Takahashi: Nonlinear ergodic theorems for semigroups of nonexpansive mappings and left ideals. Nonlinear Analysis, 26 (1996) 1411-1427.

[36] A. T. Lau, N. Shioji and W. Takahashi: Existence of nonexpansive retractions for amenable semigroups of nonexpansive mappings and nonlinear ergodic theorems in Banach spaces. J. Functional Analysis, 161 (1999) 62-75.

[37] A. T. Lau and W. Takahashi: Weak convergence and non-linear ergodic theorems for reversible semigroups of nonexpansive mappings. Pacific J. Math., 126 (1987) 277-294.

[38] A. T. Lau and W. Takahashi: Invariant means and fixed point properties for nonexpansive representations of topological semigroups. Topol. Methods Nonlinear Anal., $5(1995) 39-57$.

[39] A. T. Lau and W. Takahashi: Invariant submeans and semigroups of nonexpansive mappings on Banach spaces with normal structure. J. Functional Analysis, 142 (1996) 79-88.

[40] T. C. Lim: A fixed point theorem for families of nonexpansive mappings. Pacific J. Math., 53 (1974) 484-493.

[41] T. C. Lim: Characterization of normal structure. Proc. Amer. Math. Soc., 43 (1974) 313-319.

[42] W. R. Mann: Mean value methods in iteration. Proc. Amer. Math. Soc., 4 (1953) 506-510.

[43] B. Martinet: Regularisation, d'inèquations variationelles par approximations succesives. Revue Francaise d'Informatique et de Recherche Operationelle, (1970) 154-159.

[44] T. Mitchell: Topological semigroups and fixed points. Illinois J. Math., 14 (1970) 630-641.

[45] T. Mitchell: Fixed points of reversible semigroups of nonexpansive mappings. Kódai Math. Sem. Rep., 22 (1970) 322-323.

[46] N. Mizoguchi and W. Takahashi: On the existence of fixed points and ergodic retractions for Lipschitzian semigroups in Hilbert spaces. Nonlinear Analysis, 14 (1990) $69-80$.

[47] J. J. Moreau: Proximité et dualité dans un espace Hilbertien. Bull. Soc. Math., France, 93 (1965) 273-299.

[48] Z. Opial: Weak convergence of the sequence of successive approximations for nonexpansive mappings. Bull. Amer. Math. Soc., 73 (1967) 591-597.

[49] S. Reich: Weak convergence theorems for nonexpansive mappings in Banach spaces. J. Math. Anal. Appl., 67 (1979) 274-276.

[50] S. Reich: Strong convergence theorems for resolvents of accretive operators in Banach spaces. J. Math. Anal. Appl., 75 (1980) 287-292.

[51] B. E. Rhoades: Comments on two fixed point iteration methods. J. Math. Anal. Appl., 56 (1976) 741-750.

[52] R. T. Rockafellar: Monotone operators and the proximal point algorithm. SIAM J. Control Optim., 14 (1976) 877-898.

[53] G. Rodé: An ergodic theorem for semigroups of nonexpansive mappings in a Hilbert space. J. Math. Anal. Appl, 85 (1982) 172-178.

[54] J. Schu: Weak and strong convergence to fixed points of asymptotically nonexpansive mappings. Bull. Austral. Math. Soc., 43 (1991) 153-159. 
[55] T. Shimizu and W. Takahashi: Strong convergence to common fixed points of families of nonexpansive mappings. J. Math. Anal. Appl., 211 (1997) 71-83.

[56] N. Shioji and W. Takahashi: Strong convergence of approximated sequence for nonexpansive mappings in Banach spaces. Proc. Amer. Math. Soc., 125 (1997) 3641-3645.

[57] N. Shioji and W. Takahashi: Strong convergence theorems for asymptotically nonexpansive semigroups in Hilbert spaces. Nonlinear Analysis, 34 (1998) 87-99.

[58] N. Shioji and W. Takahashi: Strong convergence theorems for asymptotically nonexpansive semigroups in Banach spaces. J. Nonlinear Convex Anal., to appear.

[59] N. Shioji,W. Takahashi and N. Tsukiyama: A strong convergence theorem for nonexpansive mappings by the Ishikawa iteration process. to appear.

[60] T. Suzuki and W. Takahashi: On weak convergence to fixed points of nonexpansive mappings in Banach spaces. In W. Takahashi and T. Tanaka (eds.):Nonlinear Analysis and Convex Analysis (World Scientific, 1999) 341-347.

[61] G. Rodé: An ergodic theorem for semigroups of nonexpansive mappings in a Hilbert space. J. Math. Anal. Appl., 85 (1982) 172-178.

[62] W. Takahashi: Fixed point theorem for amenable semigroups of non-expansive mappings. Kódai Math. Sem. Rep., 21 (1969) 383-386.

[63] W. Takahashi: Recent results in fixed point theory. SEA Bull. Math., 4 (1981) 59-85.

[64] W. Takahashi: A nonlinear ergodic theorem for an amenable semigroup of nonexpansive mappings in a Hilbert space. Proc. Amer. Math. Soc., 81 (1981) 253-256.

[65] W. Takahashi: Fixed point theorems for families of nonexpansive mappings on unbounded sets. J. Math. Soc. Japan, 36 (1984) 543-553.

[66] W. Takahashi: A nonlinear ergodic theorem for a reversible semigroup of nonexpansive mappings in a Hilbert space. Proc. Amer. Math. Soc., 97 (1986) 55-58.

[67] W. Takahashi: Nonlinear Functional Analysis (Japanese) . (Kindaikagakusha, Tokyo, 1988).

[68] W. Takahashi: Fixed point theorem and nonlinear ergodic theorem for nonexpansive semigroups without convexity. Can. J. Math., 44 (1992) 880-887.

[69] W. Takahashi: Fixed point theorems and nonlinear ergodic theorems for nonlinear semigroups and their applications. Nonlinear Analysis, 30 (1997) 1283-1293.

[70] W. Takahashi: Fan's existence theorem for inequalities concerning ocnvex functions and its applications. In S. Simons and B. Ricceri(eds.): Minimax Theory and Applications (Kluwer Academic Publishers, 1998) 241-260.

[71] W. Takahashi and D. H. Jeong: Fixed point theorem for nonexpansive semigroups on Banach space. Proc. Amer. Math. Soc., 122 (1994) 1175-1179.

[72] W. Takahashi and G. E. Kim: Approximating fixed points of nonexpansive mappings in Banach spaces. Math. Japonica, 48 (1998) 1-9.

[73] W. Takahashi and J. Y. Park: On the asymptotic behavior of almost orbits of commutative semigroups in Banach spaces. In B. L. Lin and S. Simons (eds.): Nonlinear and Convex Analysis, Lecture Notes in Pure and Appl. Math. (Marcel Dekker, Inc., New York, 1987) 271-293.

[74] W. Takahashi and K. Shimoji: Convergence theorems for nonexpansive mappings and feasibility problems. Mathematical and Computer Modelling, to appear.

[75] W. Takahashi and T. Tamura: Limit theorems of operators by convex combinations of nonexpansive retractions in Banach spaces. J. Approximation Theory, 91 (1997) 386-397.

[76] W. Takahashi and T. Tamura: Convergence theorems for a pair of nonexpansive mappings. J. Convex Analysis, 5 (1998) 45-56.

[77] W. Takahashi and Y. Ueda: On Reich's strong convergence theorems for resolvents of 
accretive operators. J. Math. Anal. Appl., 104 (1984) 546-553.

[78] K. K. Tan and H. K. Xu: Approximating fixed points of nonexpansive mappings by the Ishikawa iteration process. J. Math. Anal. Appl., 178 (1993) 301-308.

[79] K. K. Tan and H. K. Xu: Continuous representation of semitopological semigroup as nonexpansive mappings on Banach space. to appear.

[80] R. Wittmann: Approximation of fixed points of nonexpansive mappings. Arch. Math., 58 (1992) 486-491.

[81] H. K. Xu: Inequalities in Banach spaces with applications. Nonlinear Analysis, 16 (1991) 1127-1138.

Wataru Takahashi

Department of Mathematical and Computing Sciences Tokyo Institute of Technology

Oh-okayama, Meguro-ku, Tokyo 152-8552, Japan

E-mail: wataru@is.titech.ac.jp 